\title{
Non-hotspot PIK3CA mutations are more frequent in CLOVES than in common or combined lymphatic malformations
}

Pascal Brouillard ${ }^{1 \dagger}$, Matthieu J. Schlögel ${ }^{1 \dagger}$, Nassim Homayun Sepehr ${ }^{1}$, Raphaël Helaers ${ }^{1}$, Angela Queisser ${ }^{1}$, Elodie Fastré ', Simon Boutry' , Sandra Schmitz 2,3,4, Philippe Clapuyt ${ }^{2,3,4}$, Frank Hammer 2,3,4, Anne Dompmartin ${ }^{5}$, Annamaria Weitz-Tuoretmaa ${ }^{6}$, Jussi Laranne ${ }^{7}$, Louise Pasquesoone ${ }^{8}$, Catheline Vilain ${ }^{9}$, Laurence M. Boon ${ }^{1,3,4}$ and Miikka Vikkula ${ }^{1,3,4,10^{*}}$ (1)

\begin{abstract}
Background: Theragnostic management, treatment according to precise pathological molecular targets, requests to unravel patients' genotypes. We used targeted next-generation sequencing (NGS) or digital droplet polymerase chain reaction (ddPCR) to screen for somatic PIK3CA mutations on DNA extracted from resected lesional tissue or lymphatic endothelial cells (LECs) isolated from lesions. Our cohort $(n=143)$ was composed of unrelated patients suffering from a common lymphatic malformation (LM), a combined lymphatic malformation [lymphatico-venous malformation (LVM), capillaro-lymphatic malformation (CLM), capillaro-lymphatico-venous malformation (CLVM)], or a syndrome [CLVM with hypertrophy (Klippel-Trenaunay-Weber syndrome, KTS), congenital lipomatous overgrowth-vascular malformations-epidermal nevi -syndrome (CLOVES), unclassified PIK3CA-related overgrowth syndrome (PROS) or unclassified vascular (lymphatic) anomaly syndrome (UVA)].

Results: We identified a somatic PIK3CA mutation in resected lesions of 108 out of 143 patients (75.5\%). The frequency of the variant allele ranged from 0.54 to $25.33 \%$ in tissues, and up to $47 \%$ in isolated endothelial cells. We detected a statistically significant difference in the distribution of mutations between patients with common and combined LM compared to the syndromes, but not with KTS. Moreover, the variant allele frequency was higher in the syndromes.

Conclusions: Most patients with an common or combined lymphatic malformation with or without overgrowth harbour a somatic PIK3CA mutation. However, in about a quarter of patients, no such mutation was detected, suggesting the existence of (an)other cause(s). We detected a hotspot mutation more frequently in common and combined LMs compared to syndromic cases (CLOVES and PROS). Diagnostic genotyping should thus not be limited to PIK3CA hotspot mutations. Moreover, the higher mutant allele frequency in syndromes suggests a wider distribution in patients' tissues, facilitating detection. Clinical trials have demonstrated efficacy of Sirolimus and Alpelisib in treating patients with an LM or PROS. Genotyping might lead to an increase in efficacy, as treatments could be more targeted, and responses could vary depending on presence and type of PIK3CA-mutation.
\end{abstract}

\footnotetext{
*Correspondence: miikka.vikkula@uclouvain.be

${ }^{\dagger}$ Pascal Brouillard and Matthieu J. Schlögel have contributed equally to this work

${ }^{1}$ Human Molecular Genetics, de Duve Institute, University of Louvain, Avenue Hippocrate 74 (+5), bte B1.74.06, 1200 Brussels, Belgium Full list of author information is available at the end of the article
} permits use, sharing, adaptation, distribution and reproduction in any medium or format, as long as you give appropriate credit to the original author(s) and the source, provide a link to the Creative Commons licence, and indicate if changes were made. The images or other third party material in this article are included in the article's Creative Commons licence, unless indicated otherwise in a credit line to the material. If material is not included in the article's Creative Commons licence and your intended use is not permitted by statutory regulation or exceeds the permitted use, you will need to obtain permission directly from the copyright holder. To view a copy of this licence, visit http://creativecommons.org/licenses/by/4.0/. The Creative Commons Public Domain Dedication waiver (http://creativeco mmons.org/publicdomain/zero/1.0/) applies to the data made available in this article, unless otherwise stated in a credit line to the data. 
Keywords: Lymphatic malformation, Isolated, Gene, Mutation, Somatic, PI3K, Epidemiology, Theragnostic, Allele, Frequency

\section{Background}

Vascular malformations are usually congenital and localized. They slowly develop with the growth of the child. Treatments are mostly limited to laser, sclerotherapy, embolization and surgical resection. A malformation can affect any part of the lympho-vascular system (Fig. 1). A pure malformation affects only one compartment, e.g. a common lymphatic malformation (LM), whereas combined vascular malformations associate at least two components in the same lesion, e.g. a capillaro-lymphatic malformation [1]. Patients with isolated lesions have no other associated signs, whereas syndromic-forms affect at least one other organ.

In the princeps study in 2009, it was demonstrated that somatic genetic mutations are associated with sporadically occurring vascular anomalies: isolated venous malformations (VM) were discovered to have somatic TIE2/ TEK mutations [2]. Since then, the etiopathogenesis of various isolated and combined vascular malformations

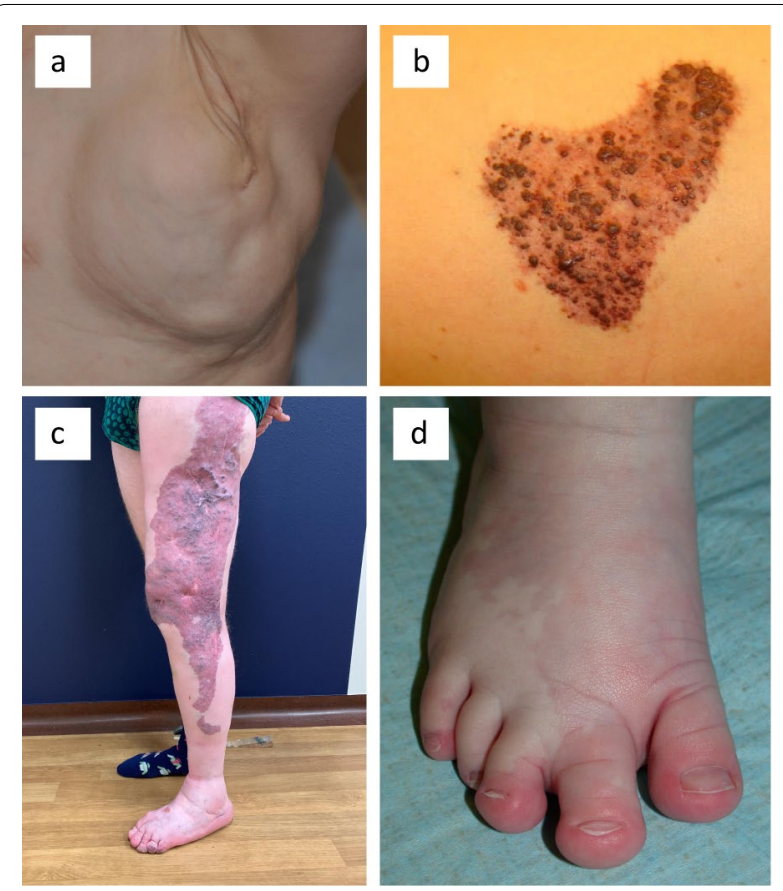

Fig. 1 Clinical characteristics of representative patients. a Mixed LM of left thorax (mutation p.Glu545Lys at $5 \%$ by NGS). b CLVM of left thorax with visible lymphatic vesicles (mutation p.His1047Arg at $5 \%$ by NGS and $5 \%$ by ddPCR). c Unclassified PROS (mutation p.Cys420Arg at 2\% by NGS). d Macrodactyly and syndactyly 2-3 with small sandal gap and CM of right foot of a CLOVES syndrome patient has been unravelled [3, 4]. One of the genes identified to be mutated in vascular malformations encodes the phosphatidylinositol-4,5-bisphosphate 3-kinase catalytic subunit alpha (PIK3CA, also known as p110 $\alpha$ ). Mutations were first identified in rare syndromic patients with Congenital Lipomatous Overgrowth, Vascular malformations, Epidermal nevi, and Skeletal/spinal abnormalities and/or scoliosis (CLOVES) [5]. Subsequently, PIK3CA mutations were implicated in common LM (43 out of 47 lesions) [6-8], and isolated VM without a TIE2/TEK mutation (27 out of 50 lesions) [9]. Somatic/mosaic PIK3CA mutations have also been implicated in variable syndromic phenotypes that can associate vascular anomalies with hypertrophy, leading to name this spectrum "PIK3CA-Related Overgrowth Syndrome" or PROS [10]. It includes CLOVES, Fibroadipose Hyperplasia $(\mathrm{FH})$, Fibroadipose infiltrating lipomatosis, Hemihyperplasia multiple lipomatosis (HHML), Klippel-Trenaunay syndrome (KTS), Megalencephaly-Capillary Malformation-Polymicrogyria syndrome (MCAP), Macrodactyly, Hemimegalencephaly, and Muscle hemihyperplasia [10].

PIK3CA is considered as an oncogene [11]. The encoded $\mathrm{p} 110 \alpha$ subunit contains the adapter-binding domain $(\mathrm{ABD})$, the Ras-binding domain (RBD), the C2-PI3K-type domain $(\mathrm{C} 2)$, the helical domain $(\mathrm{H})$ and the kinase domain (K) (Fig. 2) [12]. Mutations that spread over the five functional domains have been implicated in many cancers, with positions c.1624G (p.E542), c.1633G (p.E545) and c.3140A (p.H1047) as hotspots. Similar mutations have been found in small series of lymphatic and vascular malformations.

In this study, we analysed for the presence of a PIK3CA mutation in a large cohort of lymphatic malformations, including common LM, lymphatico-venous malformation (LVM), capillaro-lymphatic malformation (CLM), capillaro-lymphatico-venous malformation (CLVM), unilateral capillaro-lymphatico-venous malformation with hypertrophy (KTS), CLOVES syndrome, unclassified PROS and unclassified vascular anomaly syndrome (UVA). This study contains the largest cohort studied so far for isolated lymphatic malformations. We analysed the prevalence, distribution and allele frequency of PIK3CA mutations in these phenotypes and evaluated for any genotype-phenotype correlation. This study provides firm epidemiological data, essential for the design of diagnostic testing, prospective clinical trials and drug development. 


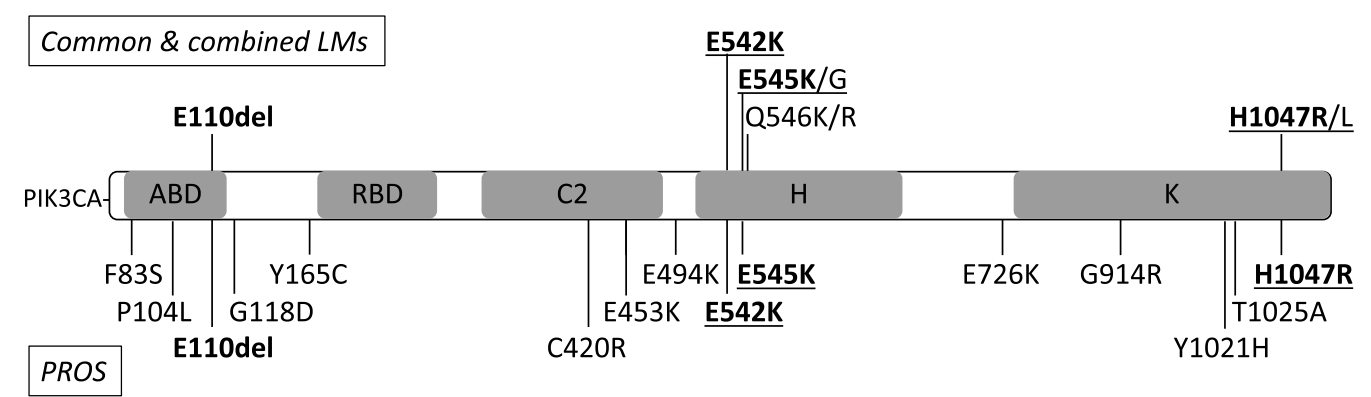

Fig. 2 PIK3CA protein (Human, 1068 aa) and mutations. Top, mutations found in common and combined lymphatic malformations (LM, LVM, CLVM). Bottom, mutations found in patients with PROS (KTS, CLOVES, unclassified PROS). See distribution in Table 1. Shared mutations in bold. Hotspot mutations underlined. ABD, p85a-binding domain; RBD, Ras-binding domain; C2, C2-PIK3C-type domain; H, helical domain; K, kinase domain

\section{Results}

To assess the contribution of PIK3CA mutations in common, combined and syndromic LM (Table 1), we screened DNA extracted from frozen tissues or isolated cells from 143 patients: 105 common LM, 3 LVM, 1 CLM, 7 CLVM, 4 KTS, 14 CLOVES, 7 PROS and 2 UVA. Within the mutated lesions, the variant allele frequency (VAF) varied strongly: from 0.54 to $25.33 \%$ for NGS-based analyses, and from $0.28 \%$ (14 SPD) to $22.7 \%$ for ddPCR-based analyses. The median VAF was $4.04 \%$ (STDEV $=4.57 \%$ ), with $60 \%$ of patients having $\leq 5 \%$ VAF. In addition, we had 8 common LMs inconclusive for the presence of hotspot mutations (5 to 9 mutant reads, but only $0.24-0.52 \%$ VAF, not confirmed by ddPCR due to lack of DNA).

PIK3CA mutations were found in 78/105 common LM (74.3\%), 3/3 LVM (100\%), 6/7 CLVM (85.7\%), 4/4 KTS (100\%), 12/14 CLOVES (85.7\%) and 5/7 PROS (71.4\%) (Table 1). No PIK3CA mutation was detected in the unique CLM and the two UVA tested. Out of the 108 mutations detected, $92(85.1 \%)$ were at hotspot positions: 31 p.E542K $(c .1624 G>A), 34$ p.E545K $(c .1633 G>A), 1$ p.E545G $(c .1634 A>G), 23$ p.H1047R $(c .3140 A>G)$ and 3 p.H1047L $(c .3140 A>T)$. The rest was covered by 14 distinct non-hotspot mutations in 16 samples (Table 1). We also isolated primary cells from two resected tissues (one LM and one CLVM), to locate the population harbouring the mutation (Table 2). Strong enrichment of the somatic mutation was observed within the isolated endothelial cells (ECs) and especially lymphatic ECs.

We detected a statistically significant difference in the distribution of hotspot mutations and non-hotspot mutations between common and combined LM compared to the syndromes $\left(p\right.$ value $\left.=1.329 \times 10^{-7}\right)$, to CLOVES $\left(p\right.$ value $\left.=1.441 \times 10^{-5}\right)$ or to unclassified PROS $(p$ value $=1.207 \times 10^{-4}$ ), but not to KTS (Fig. 3 and Table 3). Our meta-analysis of data collected from published reports detected a similar but weaker difference for CLOVES ( $p$ value $=0.0023)[6-8,10,13,14]$. When combining our data with the literature, the mutation distribution difference was significant between common and combined LMs versus PROS ( $p$ value $=7.742 \times 10^{-5}$ ) and versus CLOVES ( $p$ value $=5.575 \times 10^{-7}$ ). All $p$ values adjusted for multiple testing remained significant (Table 3). As earlier reports focussed on hotspot screenings, it probably explains the weaker association in the literature data. Out of the altogether 367 patients reported by us and others, $54(14.7 \%)$ are PIK3CA-negative: 38 LM, 1 CLM, 3 CLVM, 1 KTS, 4 CLOVES, 5 PROS and 2 UVA. Some of these could just be under the threshold of detection.

We also studied variability of VAF in different samples of the same patient. We had 16 patients for which 2, 3 or 4 samples were analysed (Table 4). A mutation was found in 14 . The detected allele frequencies had a mean average of $5.17 \%$, with an SD of $2.36 \%$. For each patient, the same mutation was identified in all tissues. The two patients without an identified somatic mutation, were each negative in 3 different samples.

Overall, VAF varied from 0.54 to $25.33 \%$ between different phenotypes (Table 1). There was a statistically highly significant difference in VAF between common and combined LMs versus syndromes (PROS) ( $p$ value $=1.425 \times 10^{-4}$, Fig. 4a) and versus CLOVES $(p$ value $=6.510 \times 10^{-5}$, Fig. $4 \mathrm{~b}$ ), as well as between common LMs versus syndromes (PROS) $\left(p\right.$ value $=6.765 \times 10^{-5}$, Fig. 4c) and versus CLOVES ( $p$ value $=5.290 \times 10^{-4}$, Fig. $4 d$ ). There was no statistically significant difference between the other entities (Additional file 1: Table S1).

The VAF for hotspot mutations varied between 0.54 and $22.19 \%(\mathrm{n}=92$, median: $3.88 \%, \mathrm{SD}=3.72)$, whereas for the non-hotspot mutations it varied between 1 and 25.33\% $(\mathrm{n}=16$, median $=8.71 \%$, $\mathrm{SD}=7.27$ ). There was no statistically significant 
Table 1 Patients cohort and PIK3CA mutations per pathology

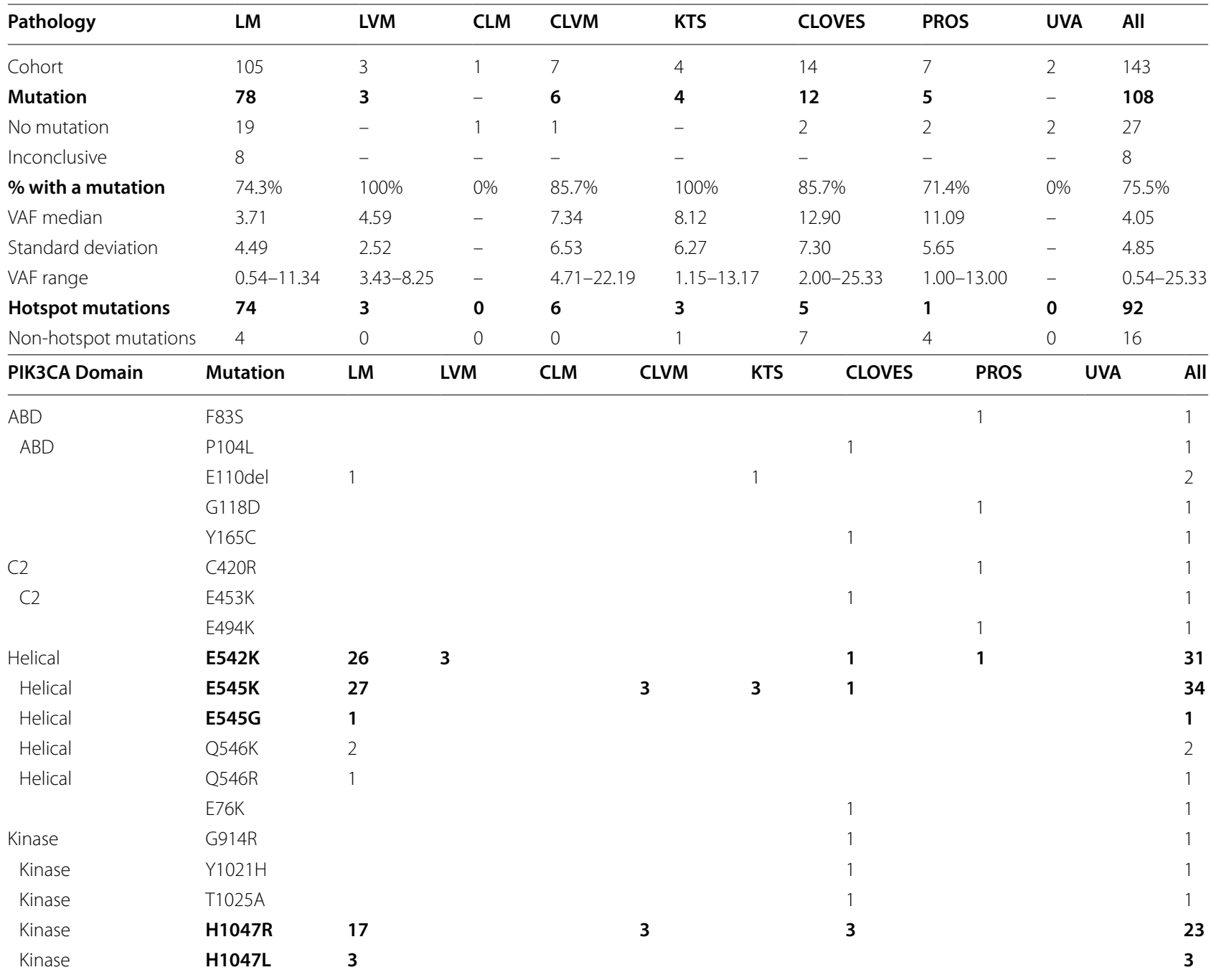

Bold: hotspot mutations. Protein domains based on https://www.uniprot.org/uniprot/P42336: ABD, p85a-binding domain (amino acids 16-105); C2, C2-PIK3C-type domain (330-487); Helical, Helical domain (517-694); Kinase, Kinase domain (797-1068). See also Fig. 2

Table 2 PIK3CA variants allele frequencies in tissues and isolated cells

\begin{tabular}{llll}
\hline Disease & Sample type & Mutation & VAF (\%) \\
\hline CLVM & Tissue & p.His1047Arg & 5 \\
& CD31-positive cells (EC) & p.His1047Arg & 47 \\
& CD31-negative cells & p.His1047Arg & 0 \\
LM & Tissue & p.Glu542Lys & 11 \\
& Unselected cells & p.Glu542Lys & 4 \\
CD34-positive cells (BEC) & p.Glu542Lys & 17 \\
& CD31-positive and CD34-nega- & p.Glu542Lys & 32 \\
tive cells (LEC) & & \\
& CD31- and CD34-negative cells & p.Glu542Lys & 0 \\
& Fibroblasts & p.Glu542Lys & 0
\end{tabular}

$\mathrm{VAF}$, variant allele frequency; $\mathrm{EC}$, endothelial cell; $\mathrm{BEC}$, blood endothelial cell; LEC, lymphatic endothelial cell difference between these two groups $(p$ value $=0.0844)$ (Fig. 4e) or with individual hotspots (Fig. 4f, Additional file 1: Table S1). Finally, we analysed associations between VAF (Fig. 4g-i and Additional file 1: Table S1) or mutations (hotspot vs non-hotspot or presence vs absence) in regard to LM localization (Table 5), size (Table 6) or cystic structure (Table 7). There was no statistically significant difference identified in adjusted $p$ values, except that a mutation is less often detected in small lesions $(p$ value $=0.0129)$ (Table 6)

\section{Discussion}

We used deep sequencing and ddPCR to study a series of lymphatic malformations. Variant allele frequency ranged from 0.54 to $25.33 \%$, suggesting that the most 


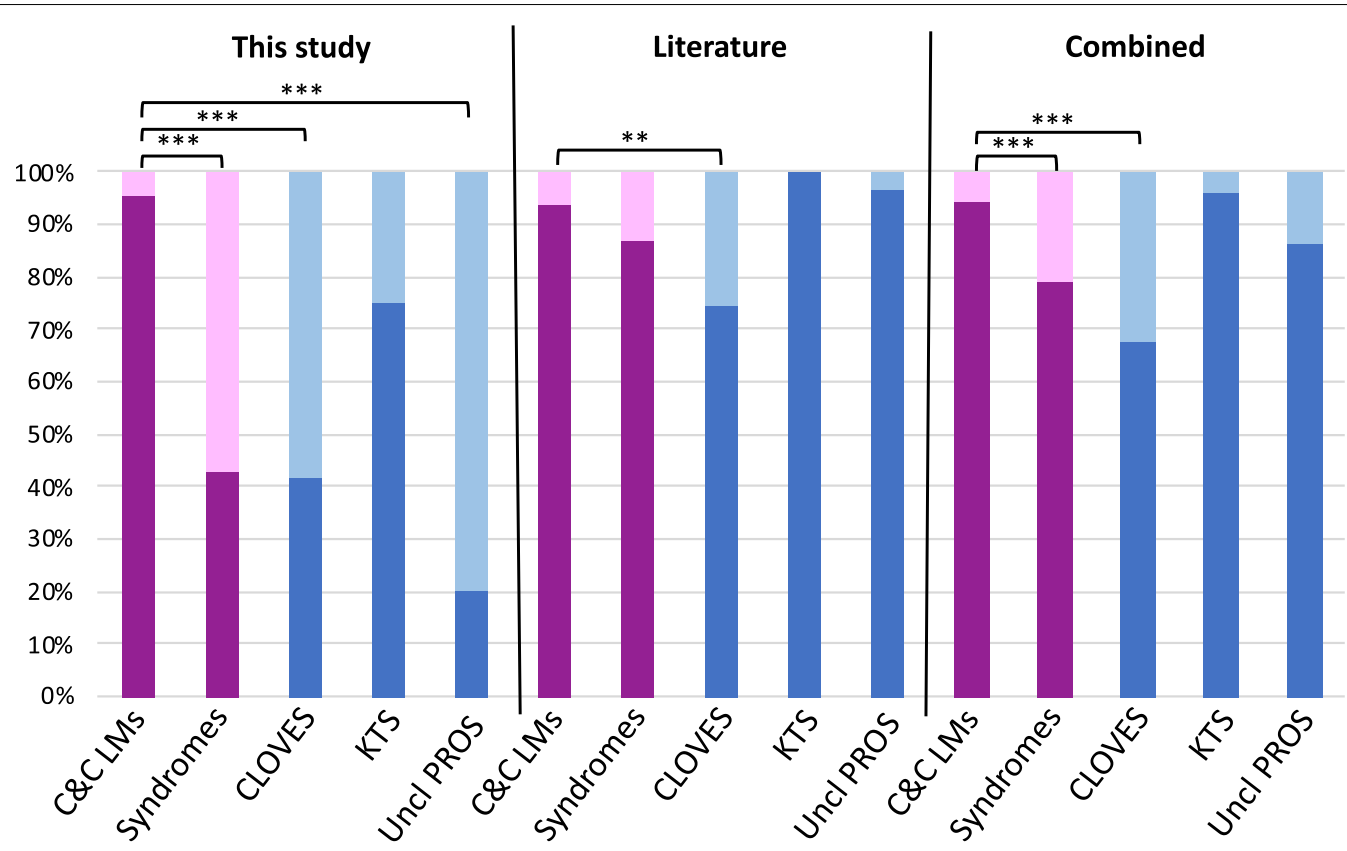

Fig. 3 Genotype distribution of PIK3CA mutations between patients with common and combined LMs or PROS. Distribution of hotspot mutations (dark colours, affecting amino acid positions 542, 545 or 1047) and non-hotspot mutations (light colours, all other amino acid positions) in patient cohorts. C\&C LMs (Common and combined LMs) includes LM, LVM and CLVM. PROS, includes CLOVES, KTS and unclassified (Uncl) PROS (shown separately in blue). Literature: from references $[6-8,10,13,14]$. Combined: this study and the literature. See Table 3 for values. ${ }^{* * *} p$ value $<0.001 .{ }^{* *} p$ value $<0.01$

Table 3 Comparison of the mutation types between the different cohorts

\begin{tabular}{|c|c|c|c|c|c|c|c|c|}
\hline & $\begin{array}{l}\text { Number of } \\
\text { patients }\end{array}$ & Pathology & Hotspot & Non-hotspot & Fisher's Exact Test & $p$ value & Adjusted $p$ value & Significance \\
\hline \multirow[t]{5}{*}{ This study } & $\mathrm{n}=87$ & C\&C LMs & 83 & 4 & & & & \\
\hline & $n=21$ & PROS & 9 & 12 & C\&C LMs vs PROS & $1.329 \mathrm{e}-07$ & $1.5948 \mathrm{e}-06$ & $* * *$ \\
\hline & 12 & CLOVES & 5 & 7 & C\&C LMS VS CLOVES & $1.441 e-05$ & $5.764 e-05$ & $* * *$ \\
\hline & 4 & KTS & 3 & 1 & C\&CLMs vs KTS & 0.2055 & 0.274 & \\
\hline & 5 & UndPROS & 1 & 4 & C\&C LMS vs Und PROS & 0.0001207 & 0.0002896 & $* * *$ \\
\hline \multirow[t]{5}{*}{ Literature } & $n=107$ & C\&C LMs & 100 & 7 & & & & \\
\hline & $\mathrm{n}=98$ & PROS & 85 & 13 & C\&C LMs vs PROS & 0.1562 & 0.2343 & \\
\hline & 47 & CLOVES & 35 & 12 & C\&C LMS VS CLOVES & 0.002331 & 0.004662 & $* *$ \\
\hline & 20 & KTS & 20 & 0 & C\&C LMs vs KTS & 0.5955 & 0.7146 & \\
\hline & 31 & UndPROS & 30 & 1 & C\&C LMs vs Und PROS & 0.6832 & 0.7453 & \\
\hline \multirow[t]{5}{*}{ Combined } & $n=194$ & C\&C LMs & 183 & 11 & & & & \\
\hline & $n=119$ & PROS & 94 & 25 & C\&C LMs vs PROS & 7.742e-05 & $2.232 \mathrm{e}-04$ & $* * *$ \\
\hline & 59 & CLOVES & 40 & 19 & C\&C LMS Vs CLOVES & $5.575 e-07$ & $3.345 e-06$ & $* * *$ \\
\hline & 24 & KTS & 23 & 1 & C\&CLMS vs KTS & 1 & 1 & \\
\hline & 36 & UndPROS & 31 & 5 & C\&C LMs vs Und PROS & 0.1434 & 0.2343 & \\
\hline
\end{tabular}

C\&C (common and combined) LMs: LM, LVM, CLVM. PROS: includes CLOVES, KTS and unclassified (Uncl) PROS. Detailed values of PROS subgroups are given in italic. Literature: from references $[6-8,10,13,14]$. Combined: this study and the literature. ${ }^{* *} p$ value $<0.01 ;{ }^{* * *} p$ value $<0.001$. The distributions between hotspot and non-hotspot are shown in Fig. 3

clonal surgical resections contained up to $50 \%$ of heterozygous mutant cells. In contrast, in the least clonal lesion detected, the frequency was as low as $1 \%$ of cells. VAF in common and combined LMs had a median of 
Table 4 Patients with multiple samples analysed

\begin{tabular}{|c|c|c|c|c|c|c|}
\hline Sequenced samples & Common code & Pathology & Mutation & VAF (\%) & $\begin{array}{l}\text { Average VAF } \\
\text { (\%) }\end{array}$ & SD VAF (\%) \\
\hline VA-10 & VA-10 & LM & E545G & 10.01 & 8.28 & 2.45 \\
\hline VA-47.pKPT & & & E545G & 6.55 & & \\
\hline VA-1124.pKPT & VA-1124 & CLOVES & E453K & 13.51 & 8.64 & 6.89 \\
\hline VA-1133.pKPT & & & E453K & 3.76 & & \\
\hline VA-1126.pKPT & VA-1126 & CLOVES & - & - & - & - \\
\hline VA-1126.pRASO2 & & & - & - & & \\
\hline VA-1126-T.pCMAVM & & & - & - & & \\
\hline VA-1158-T.pKPT & VA-1158-T & CLOVES & E545K & 12.16 & 8.66 & 4.17 \\
\hline VA-1158-T-1.pTVPP & & & E545K & 9.77 & & \\
\hline VA-1158-T-2.pTVPP & & & E545K & 4.05 & & \\
\hline VA-230.pKPT & VA-230 & LM & H1047L & 2.52 & 2.50 & 0.04 \\
\hline VA-298.pKPT & & & H1047L & 2.47 & & \\
\hline VA-24.pKPT & VA-24 & LM & - & - & - & - \\
\hline VA-240.pKPT & & & - & - & & \\
\hline VA-986.pKPT & & & - & - & & \\
\hline VA-312.pKPT & VA-312 & LM & E110delE & 1.06 & 1.90 & 0.82 \\
\hline VA-339.pKPT & & & E110delE & 1.95 & & \\
\hline VA-403.pKPT & & & E110delE & 2.70 & & \\
\hline VA-364.pKPT & VA-364 & LM & E542K & 1.11 & 2.74 & 2.30 \\
\hline VA-421.pKPT & & & E542K & 4.36 & & \\
\hline VA-50.pKPT & VA-50 & LM & E542K & 4.95 & 6.56 & 2.28 \\
\hline VA-790.pKPT & & & E542K & 8.17 & & \\
\hline VA-528.pKPT & VA-528 & LM & E545K & 4.3 & 4.93 & 2.39 \\
\hline VA-714.pKPT & & & E545K & 6.24 & & \\
\hline VA-830.pKPT & & & E545K & 7.32 & & \\
\hline VA-913.pKPT & & & E545K & 1.87 & & \\
\hline VA-711.pKPT & VA-711 & $L M$ & H1047R & 5.66 & 8.33 & 3.77 \\
\hline VA-829.pKPT & & & H1047R & 10.99 & & \\
\hline VA-756.pKPT & VA-756 & LVM & E542K & 8.25 & 7.07 & 1.67 \\
\hline VA-979.pKPT & & & E542K & 5.89 & & \\
\hline VA-1037.pKPT & VA-886 & LM & Q546R & 2.16 & 3.06 & 1.27 \\
\hline VA-886.pKPT & & & Q546R & 3.96 & & \\
\hline VA-868-T.pRASO2 & VA-868 & LM & E542K & 2.11 & 3.78 & 2.36 \\
\hline VA-1245.pKPT & & & E542K & 5.45 & & \\
\hline VA-869-T.pRASO2 & VA-869 & LM & E545K & 4.0 & 2.87 & 1.61 \\
\hline VA-1243.pKPT & & & E545K & 1.73 & & \\
\hline VA-1041.pKPT & VA-916 & LM & H1047R & 3.86 & 3.10 & 1.08 \\
\hline VA-916.pKPT & & & H1047R & 2.33 & & \\
\hline
\end{tabular}

$\mathrm{VAF}$, variant allele frequency; SD, standard deviation; mean of averages $=5.17 \%$; mean of $S D s=2.36 \%$

$3.50 \%(\mathrm{SD}=3.24)$; it was more than double in PROS samples $($ median $=8.78 \%, \mathrm{SD}=6.47)$. These are similar to the ranges previously reported [13, 14]. The higher mutant allele frequency detected in PROS patients suggests broader mosaicism.

We identified a statistically significant association between mutation types and phenotypes. The non-hotspot mutations had a higher frequency in CLOVES and unclassified PROS compared to common and combined LMs (Fig. 3). Similar has been noted for macrocephaly-capillary malformation (M-CM) in which PIK3CA mutations are more often non-hotspot mutations [15]. Moreover, $\mathrm{M}-\mathrm{CM}$ patients tend to be more mosaic for the mutation, which is sometimes detectable in their blood [15]. The level of mosaicism in human tissues may reflect the potential pathogenicity (the strength of downstream 


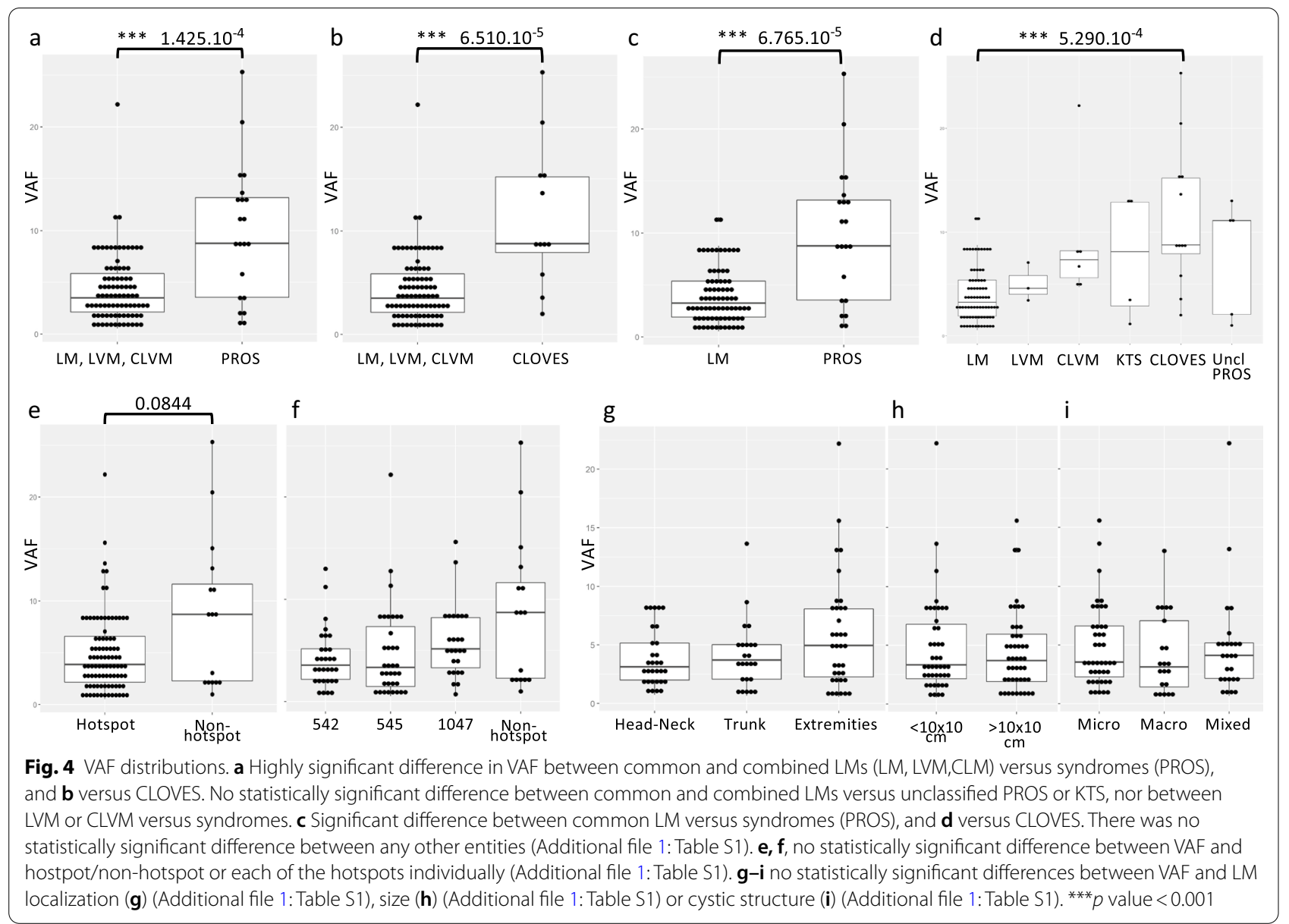

Table 5 Comparison of mutations versus localisation

\begin{tabular}{|c|c|c|c|c|}
\hline Localisation & Mutation & Hotspot & Non-hotspot & No mutation \\
\hline \multicolumn{5}{|l|}{ Input data } \\
\hline Head \& Neck & 32 & 31 & 1 & 12 \\
\hline Trunk & 25 & 23 & 2 & 11 \\
\hline Extremities & 40 & 37 & 4 & 8 \\
\hline group_1 & group_2 & $p$ value & Adjusted $p$ value & \\
\hline \multicolumn{5}{|c|}{ Fisher's exact test Mutation/no mutation } \\
\hline Head \& Neck & Trunk & 0.8068 & 0.8068 & \\
\hline Head \& Neck & Extremities & 0.3118 & 0.4677 & \\
\hline Trunk & Extremities & 0.1877 & 0.4677 & \\
\hline \multicolumn{5}{|c|}{ Fisher's exact test Hotspot/non-hotspot } \\
\hline Head \& Neck & Trunk & 0.5762 & 0.8643 & \\
\hline Head \& Neck & Extremities & 0.3732 & 0.8643 & \\
\hline Trunk & Extremities & 1 & 1 & \\
\hline
\end{tabular}

gain-of-function effects) of a given mutation. This suggests that the non-hotspot mutations that are more frequently seen in widespread PROS (such as CLOVES and
M-CM) may have weaker downstream effects and thus could occur earlier during fetal development, thereby affecting more extensive body parts. Interestingly, when 
Table 6 Comparison of mutations versus lesional size

\begin{tabular}{|c|c|c|c|c|}
\hline Size & Mutation & Hotspot & Non-hotspot & No mutation \\
\hline \multicolumn{5}{|l|}{ Input data } \\
\hline$<10 \times 10 \mathrm{~cm}$ & 46 & 45 & 1 & 23 \\
\hline$>10 \times 10 \mathrm{~cm}$ & 50 & 44 & 6 & 8 \\
\hline Unknown & 1 & 1 & 0 & 0 \\
\hline group_1 & group_2 & $p$ value & Significance & \\
\hline \multicolumn{5}{|c|}{$\begin{array}{l}\text { Fisher's exact test Mutation/no muta- } \\
\text { tion }\end{array}$} \\
\hline$<10 \times 10 \mathrm{~cm}$ & $>10 \times 10 \mathrm{~cm}$ & 0.01285 & * & \\
\hline group_1 & group_2 & $p$ value & & \\
\hline \multicolumn{5}{|c|}{ Fisher's exact test Hotspot/non-hotspot } \\
\hline$<10 \times 10 \mathrm{~cm}$ & $>10 \times 10 \mathrm{~cm}$ & 0.1134 & & \\
\hline
\end{tabular}

Table 7 Comparison of mutations versus cystic structure

\begin{tabular}{|c|c|c|c|c|}
\hline Cystic structure & Mutation & Hotspot & Non-hotspot & No mutation \\
\hline \multicolumn{5}{|l|}{ Input data } \\
\hline Microcystic & 48 & 43 & 5 & 14 \\
\hline Macrocystic & 21 & 21 & 0 & 13 \\
\hline Mixed & 28 & 26 & 2 & 4 \\
\hline Unknown & 11 & 2 & 9 & 4 \\
\hline group_1 & group_2 & $p$ value & Adjusted $p$ value & \\
\hline \multicolumn{5}{|c|}{$\begin{array}{l}\text { Fisher's exact test Mutation/no muta- } \\
\text { tion }\end{array}$} \\
\hline Microcystic & Macrocystic & 0.1536 & 0.2304 & \\
\hline Microcystic & Mixed & 0.2815 & 0.2815 & \\
\hline Macrocystic & Mixed & 0.0241 & 0.0722 & \\
\hline group_1 & group_2 & $p$ value & Adjusted $p$ value & \\
\hline \multicolumn{5}{|c|}{ Fisher's exact test Hotspot/non-hotspot } \\
\hline Microcystic & Macrocystic & 0.3132 & 0.7500 & \\
\hline Microcystic & Mixed & 1.0000 & 1.0000 & \\
\hline Macrocystic & Mixed & 0.5000 & 0.7500 & \\
\hline
\end{tabular}

Kruskal-Wallis chi-squared $=5.906, \mathrm{df}=3, p$ value $=0.1163$

KTS is defined as unilateral capillaro-lymphatico-venous malformation with hypertrophy, like in this study, it seems to have more hotspot mutations, like common and combined LMs which are usually localized.

The same amino acid substitutions are found in cancers resulting in activation of $\mathrm{p} 110 \alpha$ [11]. As vascular anomalies do not transform into malignancy, the activating p110 $\alpha$ mutations in ECs are not able to induce oncogenesis. Nevertheless, a study on 122 patients with CLOVES syndrome demonstrated a higher risk of developing a Wilms tumour (WT) [16]. This underscores the likely presence of CLOVES-associated mutations in cell types other than EC. Interestingly, two studies on patients with KTS reported that the risk of cancers in children and adults was not higher than in the general population $[17,18]$. These reports fit with our notion that especially CLOVES patients tend to have non-hotspot mutations with higher allelic frequencies. A similar risk may also be true for other PROS patients that have a wider (bilateral) phenotype.

The implication of the same PIK3CA mutation in common LM and common VM reinforces the idea that the cell type in which mutations occur influences directly the pathology [9]. LMs would be due to somatic mutations 
in lymphatic endothelial cells (LEC), whereas VMs would be due to a mutation in blood endothelial cells (BEC). The cell-type specificity of mutations was underscored by the undetectable presence of the somatic PIK3CA mutation in our LM-derived fibroblasts and our CD-31 negative cells derived from a CLVM. Moreover, there was an increased detection rate of the tissular mutation $(11 \%)$ in the LM-derived LECs (32\%) (Table 2). This reinforces the idea that the somatic mutations occur in ECs or their precursors, determining the phenotype, and contrasts with CLOVES in which fibroblasts also harbour the mutation [19].

Intercellular signalling (mutant and wild-type) and cell-matrix interactions are also likely to play an essential role in the development of vascular malformations. This is highlighted by the single mutant cell type being at very low frequency $(<1 \%)$ in the lesion. Moreover, murine modelling demonstrated that the same somatic PIK3CA mutation activated in LECs can lead to macro- or microcystic lesions, depending on the time-point of induction of expression during development and growth [20]. Intrauterine activation led to macrocystic LMs, whereas early post-natal induction led to microcystic LMs. This fits well with the concept of cell-type and time-dependent occurrence of somatic (hotspot)/mosaic (non-hotspot) mutations explaining variability in phenotype [21].

Our results indicate that PI3K-pathway inhibition could work in at least $75.5 \%$ of patients with a lymphatic malformation. In the three published prospective clinical trials using Rapamycin, an mTOR inhibitor, on patients affected by various vascular malformations, response rates were high [22-25]. In one study, all 6 patients had a somatic mutation activating PI3K signalling and the response rate was $100 \%$. In the other two studies, somatic genotyping had been performed only for a minority of the patients, yet $>85 \%$ had at least a partial response after 12 months of treatment. A PIK3CA inhibitor, BYL719 or Alpelisib, was tested on 19 patient with PROS, also showing good outcomes [26]. Similarly, the Pan-ALK inhibitor ARQ 092 (Miransertib) showed promising results on a cohort of 6 patients with PIK3CA-Related Overgrowth Syndrome [27]. These results demonstrate the positive impact of repurposing oncology drugs to patients suffering from benign vascular anomalies with a proven or likely somatic mutation that activates PI3K.

Genotyping is likely crucial in future clinical trials to increase efficacy. The high frequency of mutations identified in this study (75.5\%) suggests that LMs are mostly caused by a somatic PIK3CA mutation. However, one fourth does not seem to have a PIK3CA mutation. This could be due to low representativeness of mutant ECs in the studied tissue sample. Analysis of multiple lesional samples may increase rate of molecular diagnosis [14].
Yet, we confirmed negativity in additional samples when available ( $\mathrm{n}=2$ patients), and in the other 14 patients that were sequenced two, three, or four times, the mutation was found in all samples, albeit with variability in VAFs (Table 4).

\section{Conclusion}

In conclusion, our systematic data on a large cohort of patients with lymphatic malformations demonstrate that $75.5 \%$ of LMs, whether common, combined, or syndromic, are caused by somatic activating PIK3CA mutations. There is a statistically significant difference in mutation types between common and combined LM versus syndromic LMs (CLOVES and unclassified PROS), suggesting differential effects of the mutations. Non-hotspot mutations need to be looked for especially in the more wide-spread PROS phenotypes. Based on these and earlier data, repurposing of PI3K signalling pathway inhibitors for the treatment of LMs, whether isolated, combined or syndromic, have a sound epidemiological and pathophysiological basis.

\section{Methods \\ Aim}

Identification of prevalence of PIK3CA mutations and genotype-phenotype correlations in pure and combined lymphatic malformations.

\section{Design}

Tissues were collected from the leftover of programmed surgeries and snap-frozen in liquid nitrogen. Clinical data in regard to the phenotype (Table 1), and localization (head \& neck, trunk and extremities), size (smaller or larger than $10 \times 10 \mathrm{~cm}$ ) and cystic structure (micro, macro, mixed) of the lesions were collected (Table 8). Nine of the patients have been included in earlier reports: one KTS as patient \#5 in (9) and the associated E545K mutation in (11); five common LMs with H1047R with histology as patients \#1-5 in (22), and two LMs (patients \#2 and \#6) as well as one KTS (patient \#18) in a clinical trial (25).

Targeted next-generation sequencing (NGS) or ddPCR to screen for somatic PIK3CA mutations on DNA extracted from resected lesional tissue or lymphatic endothelial cells (LECs) isolated from lesions.

\section{DNA extraction}

DNA extraction from frozen tissues as previously described [9]. DNA was quantified using NanoDrop 8000 
Table 8 Summary of samples per cystic structure, size and localisation

\begin{tabular}{|c|c|c|c|c|c|c|c|c|c|}
\hline \multirow{2}{*}{$\begin{array}{l}\text { Cystic structure } \\
\text { Size* }\end{array}$} & \multirow[t]{2}{*}{ Mutation } & \multirow[t]{2}{*}{ Hotspot } & \multirow[b]{2}{*}{$542 \mathrm{~K}$} & \multirow[b]{2}{*}{$545 \mathrm{~K}$} & \multirow[b]{2}{*}{1047} & \multirow[t]{2}{*}{ Non-hotspot } & \multirow[t]{2}{*}{ No mutation } & \multirow[t]{2}{*}{ Total } & \multirow[t]{2}{*}{$\%$ mutation } \\
\hline & & & & & & & & & \\
\hline Microcystic & 48 & 43 & 14 & 15 & 14 & 5 & 14 & $\underline{62}$ & $77 \%$ \\
\hline$<10 \times 10 \mathrm{~cm}$ & $20(7 / 4 / 9)$ & $19(7 / 3 / 9)$ & 10 & 4 & 5 & $1(0 / 1 / 0)$ & $9(3 / 3 / 3)$ & 29 & $69 \%$ \\
\hline$>10 \times 10 \mathrm{~cm}$ & $27(6 / 10 / 11)$ & $23(5 / 9 / 9)$ & 4 & 11 & 8 & $4(1 / 1 / 2)$ & $5(0 / 2 / 3)$ & 32 & $84 \%$ \\
\hline Unknown & $1(0 / 1 / 0)$ & $1(0 / 1 / 0)$ & 0 & 0 & 1 & 0 & 0 & 1 & $100 \%$ \\
\hline Macrocystic & 21 & 21 & 9 & 10 & 2 & 0 & 13 & $\underline{34}$ & $62 \%$ \\
\hline$<10 \times 10 \mathrm{~cm}$ & $16(7 / 2 / 7)$ & $16(7 / 2 / 7)$ & 6 & 9 & 1 & 0 & $12(5 / 6 / 1)$ & 28 & $57 \%$ \\
\hline$>10 \times 10 \mathrm{~cm}$ & $5(2 / 1 / 2)$ & $5(2 / 1 / 2)$ & 3 & 1 & 1 & 0 & $1(1 / 0 / 0)$ & 6 & $83 \%$ \\
\hline Unknown & 0 & 0 & 0 & 0 & 0 & 0 & 0 & 0 & - \\
\hline Mixed & 28 & 26 & 8 & 9 & 9 & 2 & 4 & $\underline{32}$ & $88 \%$ \\
\hline$<10 \times 10 \mathrm{~cm}$ & $10(4 / 3 / 3)$ & $10(4 / 3 / 3)$ & 4 & 4 & 2 & 0 & $2(2 / 0 / 0)$ & 12 & $83 \%$ \\
\hline$>10 \times 10 \mathrm{~cm}$ & $18(6 / 4 / 8)$ & $16(6 / 4 / 6)$ & 4 & 5 & 7 & $2(0 / 0 / 2)$ & $2(1 / 0 / 1)$ & 20 & $90 \%$ \\
\hline Unknown & 0 & 0 & 0 & 0 & 0 & 0 & 0 & 0 & - \\
\hline Unknown & 11 & 2 & 0 & 1 & 1 & 9 & 4 & $\underline{15}$ & $73 \%$ \\
\hline Total & $\underline{108}$ & $\underline{92}$ & $\underline{31}$ & $\underline{35}$ & $\underline{26}$ & $\underline{16}$ & $\underline{35}$ & $\underline{143}$ & $\underline{76 \%}$ \\
\hline
\end{tabular}

*Numbers per localisation given between parenthesis: (Head\&Neck/Trunk/Extremities). See Tables 6 and 7 for globalized numbers per size and localization. Mutation numbers are subdivided in Hotspot and Non-hotspot, with Hotspot numbers further subdivided per position

(Thermo Fisher Scientific) and Qubit 2.0 (Thermo Fisher Scientific).

\section{Isolation and culturing of primary endothelial cells (EC) and primary lymphatic ECs}

Single-cell solutions were obtained from CLVM and LM tissues by digesting with $0.04 \%$ dispase (Gibco), $0.25 \%$ collagenase II (Roche) and 0.01\% DNAse I (Roche) for $1 \mathrm{~h}$ at $37^{\circ} \mathrm{C}$. Separated cells (=mixed cells) were seeded on fibronectin-coated flasks $\left(2 \mu \mathrm{g} / \mathrm{cm}^{2}\right)$ (Millipore) and grown in ECGM2 (Bio-Connect life sciences) for CLVM isolated cells and ECGM-MV2 (Bio-Connect life sciences) for LM isolated cells, both supplemented with penicillin-streptomycin. When mixed cells reached a confluency of $80 \%$, they were detached using Accutase (Sigma). Mixed CLVM cells were sorted for CD31-positive cells using Anti-CD31 MicroBeads (Miltenyi). To obtain LECs, we performed a fibroblast depletion using Anti-fibroblast MicroBeads (Miltenyi) as well as CD34positive blood EC depletion using Anti-CD34 MicroBeads (Miltenyi), followed by a CD31-positive selection using Anti-CD31 MicroBeads. All different selected cells were plated on fibronectin-coated flasks $\left(2 \mu \mathrm{g} / \mathrm{cm}^{2}\right)$. Cell morphology was confirmed using a bright field microscope (Zeiss).

\section{PIK3CA sequencing}

We designed an Ion AmpliSeq panel for targeted sequencing of the 21 coding exons of PIK3CA and ten nucleotides of all flanking introns (NM_006218.2) (http:// www.ampliseq.com). Theoretical horizontal coverage was $96.75 \%$, with 16 bp in the 5'UTR (exon 1) and 32 bp in exon 20 not covered. The panel consisted of 2 pools of primers for multiplexed PCR-amplification with Ion Ampliseq Library kit, and sequencing on an Ion Personal Genome Machine (PGM) or an ion Proton (Thermo Fisher Scientific). Reads were aligned to the human reference sequence hg19, using the Torrent Suite Server. Bam files were imported into Highlander software package (https://sites.uclouvain.be/highlander/) for analysis. We selected variants with at least 5 mutant reads representing at minimum $1 \%$ of all alleles by interrogating all positions reported with at least 4 changes in the COSMIC database (https://cancer.sanger.ac.uk/cosmic). Samples needed to have an average coverage above $500 \times$ to be considered not to contain a mutation. Mutations with a VAF below $1 \%$ but confirmed by ddPCR when DNA was available, were considered to contain a mutation.

\section{Digital droplet PCR (ddPCR)}

Digital droplet PCR (ddPCR) was used to study four known PIK3CA hotspot mutations: c.1624G $>A$ (p.E542K), c.1633G $>A \quad$ (p.E545K), $\quad$ c.3140A $>T$ (p.H1047L) and c.3140A > G (p.H1047R) (NM_006218.2). Probes were designed by Bio-Rad (Bio-Rad Laboratories). The ddPCR experiments were performed, as described by Hindson et al. [28]. DNA input was $30 \mathrm{ng}$. At most, 93 samples were run in parallel. For the analysis, we used QuantaSoft software (Version 1.7). Samples had to have at least 5 mutant single positive droplets (SPD) among a minimum of 10,000 droplets to be considered mutated. 
Nine patients with detection of mutation by ddPCR could not be tested by NGS. Their variant allele frequencies ranged from 1.6 to $15.6 \%$.

\section{Statistics}

Data were analyzed to detect differences between groups using Fisher's exact test for two categorical variables, Pairwise Wilcoxon's rank-sum test for group comparisons of continuous variables, and the KruskalWallis test for $>2$ group comparisons of continuous variables. Nonparametric tests were used due to small sample size and non-normal distributions (tested with Shapiro test). Significance of $p$ values: ${ }^{*} p \leq 0.05$, ${ }^{* * *} p \leq 0.01$, and $* * p \leq 0.001$. In order to control type I errors, multiple testing corrections were performed using Benjamini-Hochberg procedure. All analyses were performed using $\mathrm{R}$ and graphs were generated with ggplot2. Descriptive statistics were computed using groupedstats $\mathrm{R}$ package. VAF values for statistics were based on NGS results (average VAF values for patients with multiple sequencing, Table 4) or ddPCR (for 10 samples tested only by ddPCR).

\section{Supplementary Information}

The online version contains supplementary material available at https://doi. org/10.1186/s13023-021-01898-y.

Additional file 1. Statistical comparisons of data shown in Fig. 4 d,f, g, h, i.

\section{Acknowledgements}

We are grateful to all the patients for their invaluable participation. The authors thank Liliana Niculescu for secretarial help, and Audrey Debue and Fabrice Cahay for technical assistance. Five of the authors of this publication are members of the Vascular Anomalies Working Group (VASCA WG) of the European Reference Network for Rare Multisystemic Vascular Diseases (VASCERN)_Project ID: 769036

\section{Authors' contributions}

MJS, NHS and EF carried out the DNA extractions, ddPCR and NGS panel experiments. PB and MJS analysed the data. RH developed bioinformatic tools for the analysis of somatic mutations within Highlander. AQ carried out the cell biology experiments. SB performed the statistical analyses. SS, PC, FH, AD, AWT, JL, LP, CV and LMB collected medical information and blood samples. MV conceived and coordinated the study. MJS and PB drafted the manuscript. PB and $\mathrm{MV}$ helped to develop the manuscript. All authors read and approved the final manuscript.

\section{Funding}

These studies were financially supported by the Fonds de la Recherche Scientifique-FNRS Grants T.0026.14 (to MV) and T.0146.16 (to LB), the Fund Generet managed by the King Baudouin Foundation (to MV), and by la Région wallonne dans le cadre du financement de l'axe stratégique FRFS-WELBIO (to MV). P.B. is a Scientific Logistics Manager of the Genomics Platform of University of Louvain. The authors thank the Genomics Platform of University of Louvain for lonTorrent PGM Next Generation Sequencing. We also thank the National Lottery, Belgium and the Foundation against Cancer (2010-101), Belgium for their support to the Genomics Platform of University of Louvain and de Duve Institute, as well as the Fonds de la Recherche Scientifique-FNRS Equipment Grant U.N035.17 for the «Big data analysis cluster for NGS at UCL». MS and EF were doctoral students supported by the Fonds pour la Formation à la Recherche dans I'Industrie et dans I'Agriculture (FRIA).

\section{Availability of data and materials}

The datasets used and/or analysed during the current study are available from the corresponding author on reasonable request.

\section{Declarations}

Ethics approval and consent to participate

Informed consent was obtained for all patients, as approved by the ethical committee of the Medical Faculty at the University of Louvain, Brussels, Belgium (ref B403201629786) and the local committees of collaborators.

\section{Consent for publication}

All patients gave consent to publish the pictures shown in Fig. 1.

\section{Competing interest}

The authors declare no conflict of interest.

\section{Author details}

${ }^{1}$ Human Molecular Genetics, de Duve Institute, University of Louvain, Avenue Hippocrate 74 (+5), bte B1.74.06, 1200 Brussels, Belgium. ${ }^{2}$ Otolaryngology Department, Cliniques Universitaires Saint-Luc, University of Louvain, Brussels, Belgium. ${ }^{3}$ Center for Vascular Anomalies, Division of Plastic Surgery, Cliniques Universitaires Saint-Luc, University of Louvain, Brussels, Belgium. ${ }^{4}$ VASCERN VASCA European Reference Centre, Brussels, Belgium. ${ }^{5}$ Department of Dermatology, Université de Caen Basse Normandie, CHU Caen, Caen, France. ${ }^{6}$ Department of Otolaryngology, Kokkola Central Hospital, Kokkola, Finland. ${ }^{7}$ Department of Otorhinolaryngology, Head and Neck Surgery, Tampere University Hospital, Tampere, Finland. ${ }^{8}$ Service de Chirurgie Plastique Reconstructive, Hôpital Salengro, CHU de Lille, Lille, France. ${ }^{9}$ Department of Genetics, Hôpital Erasme, ULB Center of Human Genetics, Université Libre de Bruxelles, Brussels, Belgium. ${ }^{10}$ Walloon Excellence in Lifesciences and Biotechnology (WELBIO), University of Louvain, Brussels, Belgium.

Received: 2 December 2020 Accepted: 29 May 2021

Published online: 10 June 2021

\section{References}

1. Wassef M, Blei F, Adams D, Alomari A, Baselga E, Berenstein A, et al. Vascular anomalies classification: recommendations from the international society for the study of vascular anomalies. Pediatrics. 2015;136(1):e203-14.

2. Limaye N, Boon LM, Vikkula M. From germline towards somatic mutations in the pathophysiology of vascular anomalies. Hum Mol Genet. 2009;18(R1):R65-74

3. Dekeuleneer V, Seront E, Van Damme A, Boon LM, Vikkula M. Theranostic advances in vascular malformations. J Invest Dermatol. 2020;140(4):756-63.

4. Schlögel M, Brouillard P, Boon L, Vikkula M. Molecular genetics of lymphatic and complex vascular malformations. In: Lee BB, Rockson SG, editors. Lymphedema: a concise compendium of theory and practice. 2nd ed. Berlin: Springer; 2018. p. 753-64.

5. Keppler-Noreuil KM, Sapp JC, Lindhurst MJ, Parker VE, Blumhorst C, Darling T, et al. Clinical delineation and natural history of the PIK3CA-related overgrowth spectrum. Am J Med Genet A. 2014;164(7):1713-33.

6. Boscolo E, Coma S, Luks VL, Greene AK, Klagsbrun M, Warman ML, et al. AKT hyper-phosphorylation associated with PI3K mutations in lymphatic endothelial cells from a patient with lymphatic malformation. Angiogenesis. 2015;18(2):151-62.

7. Osborn AJ, Dickie P, Neilson DE, Glaser K, Lynch KA, Gupta A, et al. Activating PIK3CA alleles and lymphangiogenic phenotype of lymphatic endothelial cells isolated from lymphatic malformations. Hum Mol Genet. 2015:24(4):926-38.

8. Luks VL, Kamitaki N, Vivero MP, Uller W, Rab R, Bovee JV, et al. Lymphatic and other vascular malformative/overgrowth disorders are caused by somatic mutations in PIK3CA. J Pediatr. 2015;166(4):1048-54 e1-5.

9. Limaye N, Kangas J, Mendola A, Godfraind C, Schlogel MJ, Helaers R, et al. Somatic activating PIK3CA mutations cause venous malformation. Am J Hum Genet. 2015;97(6):914-21. 
10. Keppler-Noreuil KM, Parker VE, Darling TN, Martinez-Agosto JA. Somatic overgrowth disorders of the PI3K/AKT/mTOR pathway \& therapeutic strategies. Am J Med Genet C Semin Med Genet. 2016;172(4):402-21.

11. Samuels Y, Wang Z, Bardelli A, Silliman N, Ptak J, Szabo S, et al. High frequency of mutations of the PIK3CA gene in human cancers. Science. 2004;304(5670):554.

12. Burke JE, Perisic O, Masson GR, Vadas O, Williams RL. Oncogenic mutations mimic and enhance dynamic events in the natural activation of phosphoinositide 3-kinase p110alpha (PIK3CA). Proc Natl Acad Sci U S A. 2012;109(38):15259-64.

13. Kurek KC, Luks VL, Ayturk UM, Alomari Al, Fishman SJ, Spencer SA, et al. Somatic mosaic activating mutations in PIK3CA cause CLOVES syndrome. Am J Hum Genet. 2012;90(6):1108-15.

14. Zenner K, Cheng CV, Jensen DM, Timms AE, Shivaram G, Bly R, et al. Genotype correlates with clinical severity in PIK3CA-associated lymphatic malformations. JCl Insight. 2019;4(21):e129884.

15. Riviere JB, Mirzaa GM, O'Roak BJ, Beddaoui M, Alcantara D, Conway RL, et al. De novo germline and postzygotic mutations in AKT3, PIK3R2 and PIK3CA cause a spectrum of related megalencephaly syndromes. Nat Genet. 2012:44(8):934-40.

16. Peterman CM, Fevurly RD, Alomari Al, Trenor CC, 3rd, Adams DM, Vadeboncoeur S, et al. Sonographic screening for Wilms tumor in children with CLOVES syndrome. Pediatr Blood Cancer. 2017;64(12). https:// doi.org/10.1002/pbc.26684.

17. Blatt J, Finger M, Price V, Crary SE, Pandya A, Adams DM. Cancer risk in Klippel-Trenaunay syndrome. Lymphat Res Biol. 2019;17(6):630-6.

18. Greene AK, Kieran M, Burrows PE, Mulliken JB, Kasser J, Fishman SJ. Wilms tumor screening is unnecessary in Klippel-Trenaunay syndrome. Pediatrics. 2004;113(4):e326-9.

19. Loconte DC, Grossi V, Bozzao C, Forte G, Bagnulo R, Stella A, et al. Molecular and functional characterization of three different postzygotic mutations in PIK3CA-related overgrowth spectrum (PROS) patients: effects on PI3K/AKT/mTOR signaling and sensitivity to PIK3 inhibitors. PLOS ONE. 2015;10(4):e0123092.
20. Martinez-Corral I, Zhang Y, Petkova M, Ortsater H, Sjoberg S, Castillo SD, et al. Blockade of VEGF-C signaling inhibits lymphatic malformations driven by oncogenic PIK3CA mutation. Nat Commun. 2020;11(1):2869.

21. Van Damme A, Seront E, Dekeuleneer V, Boon LM, Vikkula M. New and emerging targeted therapies for vascular malformations. Am J Clin Dermatol. 2020;21(5):657-68.

22. Boscolo E, Limaye N, Huang L, Kang KT, Soblet J, Uebelhoer M, et al. Rapamycin improves TIE2-mutated venous malformation in murine model and human subjects. J Clin Invest. 2015;125(9):3491-504.

23. Hammer J, Seront E, Duez S, Dupont S, Van Damme A, Schmitz S, et al. Sirolimus is efficacious in treatment for extensive and/or complex slowflow vascular malformations: a monocentric prospective phase II study. Orphanet J Rare Dis. 2018;13(1):191.

24. Hammill AM, Wentzel M, Gupta A, Nelson S, Lucky A, Elluru R, et al. Sirolimus for the treatment of complicated vascular anomalies in children. Pediatr Blood Cancer. 2011;57(6):1018-24.

25. Seront E, Van Damme A, Boon LM, Vikkula M. Rapamycin and treatment of venous malformations. Curr Opin Hematol. 2019;26(3):185-92.

26. Venot Q, Blanc T, Rabia SH, Berteloot L, Ladraa S, Duong JP, et al. Targeted therapy in patients with PIK3CA-related overgrowth syndrome. Nature. 2018;558(7711):540-6.

27. Ranieri C, Di Tommaso S, Loconte DC, Grossi V, Sanese P, Bagnulo R, et al. In vitro efficacy of ARQ 092, an allosteric AKT inhibitor, on primary fibroblast cells derived from patients with PIK3CA-related overgrowth spectrum (PROS). Neurogenetics. 2018;19(2):77-91.

28. Hindson BJ, Ness KD, Masquelier DA, Belgrader P, Heredia NJ, Makarewicz AJ, et al. High-throughput droplet digital PCR system for absolute quantitation of DNA copy number. Anal Chem. 2011;83(22):8604-10.

\section{Publisher's Note}

Springer Nature remains neutral with regard to jurisdictional claims in published maps and institutional affiliations.
Ready to submit your research? Choose BMC and benefit from:

- fast, convenient online submission

- thorough peer review by experienced researchers in your field

- rapid publication on acceptance

- support for research data, including large and complex data types

- gold Open Access which fosters wider collaboration and increased citations

- maximum visibility for your research: over $100 \mathrm{M}$ website views per year

At BMC, research is always in progress.

Learn more biomedcentral.com/submissions 